\title{
Comparison of a near-infrared reflectance spectroscopy system and skin conductance measurements for in vivo estimation of skin hydration: a clinical study
}

\author{
Inga Saknite ${ }^{{ }^{*}}$, Aleksejs Zavorins ${ }^{2}$, Ilona Zablocka ${ }^{2}$, Janis Kisis ${ }^{3}$, and Janis Spigulis ${ }^{1}$ \\ ${ }^{1}$ Biophotonics Laboratory, Institute of Atomic Physics and Spectroscopy, University of Latvia, Raina Blvd. 19, \\ Riga LV-1586, Latvia \\ ${ }^{2}$ Faculty of Continuing Education, Riga Stradins University, Dzirciema Str. 16, Riga, LV-1007, Latvia \\ ${ }^{3}$ Department of Infectology and Dermatology, Riga Stradins University, Linezera Str. 3, Riga, LV-1006, Latvia \\ * e-mail: inga.saknite@lu.lv
}

\begin{abstract}
Diffuse reflectance spectroscopy system was developed for estimation of skin hydration in the near-infrared spectral range of 900-1700 nm. Experimental setup consisted of a near-infrared spectrometer, Y-type fiber optics probe with 1 detection and 6 illumination fibers, halogen-tungsten light source and a PC. By analyzing diffuse reflectance spectrum, a parameter representing skin hydration by performing baseline correction and calculating the area under the $1450 \mathrm{~nm}$ water absorption maximum is proposed. A clinical study was performed acquiring data of skin hydration of 39 patients' forearm skin. Results of the developed system are compared to results obtained by a commercial device based on skin conductance measurements. (C) 2017 Journal of Biomedical Photonics \& Engineering.
\end{abstract}

Keywords: Diffuse reflectance; near-infrared; spectroscopy; water; absorption; hydration; skin.

Paper \#3166 received 1 Mar 2017; revised manuscript received 26 Apr 2017; accepted for publication 26 Apr 2017; published online 28 Apr 2017. doi: 10.18287/JBPE17.03.010310. [Special Issue. Years in Biophotonics: 70th Anniversary of Prof. A.V. Priezzhev].

\section{References}

1. M. Egawa, M. Yanai, K. Kikuchi, and Y. Masuda, "Extended range near-infrared imaging of water and oil in facial skin,” Appl. Spectrosc. 65(8), 924-930 (2011).

2. H. Arimoto, and M. Egawa, "Water content distribution imaging of skin tissue using near-infrared camera and measurement depth analysis," Proc SPIE 8587, 85871T (2013).

3. R. H. Wilson, K. P. Nadeau, F. B. Jaworski, B. J. Tromberg, and A. J. Durkin, "Review of short-wave infrared spectroscopy and imaging methods for biological tissue characterization,” J. Biomed. Opt. 20(3), 030901 (2015).

4. J. Wang, Y.-J. Geng, B. Guo, T. Klima, B. N. Lal, J. T. Willerson, and W. Casscells, "Near-infrared spectroscopic characterization of human advanced atherosclerotic plaques," J. Am. Coll. Cardiol. 39(8), 1305$1313(2002)$.

5. P. R. Moreno, R. A. Lodder, K. R. Purushothaman, W. E. Charash, W. N. O'Connor, and J. E. Muller, "Detection of lipid pool, thin fibrous cap, and inflammatory cells in human aortic atherosclerotic plaques by near-infrared spectroscopy," Circulation 105(8), 923-927 (2002).

6. R. Nachabé, D. J. Evers, B. H. W. Hendriks, G. W. Lucassen, M. van der Voort, E. J. Rutgers, M.-J. V. Peeters, J. a Van der Hage, H. S. Oldenburg, J. Wesseling, and T. J. M. Ruers, "Diagnosis of breast cancer using diffuse optical spectroscopy from 500 to $1600 \mathrm{~nm}$ : comparison of classification methods," J. Biomed. Opt. 16(8), 087010 (2011).

7. I. Saknite, and J. Spigulis, "Determination of in vivo skin moisture level by near-infrared reflectance spectroscopy,” Proc. SPIE 9332, 93320Y (2015). 
8. R. R. Wickett, and M. O. Visscher, "Structure and function of the epidermal barrier," Am. J. Infect. Control 34(10), S98-S110 (2006).

9. K. Nolan, and E. Marmur, "Moisturizers: Reality and the skin benefits," Dermatol. Ther. 25(3), 229-233 (2012).

10. M. Egawa, and H. Tagami, "Comparison of the depth profiles of water and water-binding substances in the stratum corneum determined in vivo by Raman spectroscopy between the cheek and volar forearm skin: Effects of age, seasonal changes and artificial forced hydration," Br. J. Dermatol. 158(2), 251-260 (2008).

11. E. Proksch, and J.-M. Lachapelle, "The management of dry skin with topical emollients - recent perspectives," JDDG J. der Dtsch. Dermatologischen Gesellschaft 3(10), 768-774 (2005).

12. S. Wiedersberg, C. S. Leopold, and R. H. Guy, "Effects of Various Vehicles on Skin Hydration in vivo," Ski. Pharmacol Physiol 22(3), 128-130 (2009).

13. M. Y. Akhalaya, G. V. Maksimov, A. B. Rubin, J. Lademann, and M. E. Darvin, "Molecular action mechanisms of solar infrared radiation and heat on human skin," Ageing Res. Rev. 16, 1-11 (2014).

14. B. Cugmas, M. Bürmen, M. Bregar, F. Pernuš, and B. Likar, "Pressure-induced near infrared spectra response as a valuable source of information for soft tissue classification," J. Biomed. Opt. 18(4), 047002 (2013).

15. B. Cugmas, M. Bregar, M. Bürmen, F. Pernuš, and B. Likar, "Impact of contact pressure-induced spectral changes on soft-tissue classification in diffuse reflectance spectroscopy: problems and solutions," J. Biomed. Opt. 19(3), 037002 (2014).

16. M. Yu. Kirillin, P. D. Agrba, and V. A. Kamensky, "In vivo study of the effect of mechanical compression on formation of OCT images of human skin," J. Biophotonics 3(12), 752-758 (2010).

17. D. L. Edbrooke, C. L. Hibbert, J. M. Kingsley, S. Smith, N. M. Bright, and J. M. Quinn, "The patient-related costs of care for sepsis patients in a United Kingdom adult general intensive care unit," Crit. Care Med. 27(9), 1760-1767 (1999).

18. A. A. Gurjarpadhye, , W. C. Vogt, Y. Liu, and C. G. Rylander, "Effect of localized mechanical indentation on skin water content evaluated using OCT," Int. J. Biomed. Imaging 2011, 817250 (2011).

19. T. J. Allen, A. Hall, A. P. Dhillon, J. S. Owen, and P. C. Beard, "Spectroscopic photoacoustic imaging of lipidrich plaques in the human aorta in the 740 to $1400 \mathrm{~nm}$ wavelength range," J. Biomed. Opt. 17(6), 061209 (2012).

20. S. Nielsen, Food analysis, Springer Science \& Business Media (2014).

21. A. N. Bashkatov, E. A. Genina, V. I. Kochubey, and V. V Tuchin, "Optical properties of human skin, subcutaneous and mucous tissues in the wavelength range from 400 to $2000 \mathrm{~nm}$," J. Phys. D. Appl. Phys. 38(15), 2543-2555 (2005).

22. J. Serup, G. B. E. Jemec, and G. L. Grove, Handbook of Non-Invasive Methods and the Skin, Second Edition, CRC Press (2006).

23. M. G. Sowa, J. R. Payette, and H. H. Mantsch, "Near-infrared spectroscopic assessment of tissue hydration following surgery," J Surg Res 86(1), 62-69 (1999).

\section{Introduction}

\subsection{Near-infrared reflectance spectroscopy}

Near-infrared diffuse reflectance spectroscopy is a technique that has a potential to distinguish between different water volume fractions in skin as water has distinct absorption peaks in the near-infrared (NIR) and short-wave infrared (SWIR) spectral ranges. Thus, NIR and SWIR spectroscopy is useful and informative for distinguishing between different hydration levels in skin which is very interesting for the cosmetic industry, as well as for dermatologists. There are three main areas of research regarding diffuse reflectance spectroscopy in the NIR and SWIR spectral ranges: 1) integrating sphere based measurements for estimating ex vivo tissue absorption $\mu_{a}$ and scattering $\mu_{s}$ coefficients; 2) fiber probe based "point" measurements to acquire reflectance measurements collected over small localized tissue volumes both in vivo and ex vivo; 3) imaging measurements both in vivo [1-2] and ex vivo that offer non-contact measurements of a larger area of tissues [3]. Previous studies based on fiber probe reflectance spectroscopy method have shown NIR and SWIR spectroscopy applications for distinguishing between $e x$ vivo vulnerable and stable atherosclerotic plaques by estimating lipid-to-protein ratios [4-5], as well as distinguishing between ex vivo normal and cancerous breast tissue by estimating water and lipid volume fractions [6].

In our previous work [7] we presented a method to estimate skin hydration changes over a short period after application of different moisturizing creams based on near-infrared spectroscopy in the spectral range of 900$1700 \mathrm{~nm}$. We proposed a skin hydration parameter, calculated as difference in absorbance between local water absorption maximum $(1450 \mathrm{~nm})$ and local water absorption minimum $(1300 \mathrm{~nm})$. It was possible to see hydration parameter changes over time after a moisturizing cream was applied on skin surface, however, we concluded that the sensitivity of the method should be improved. 


\subsection{Skin hydration}

Skin is the largest organ of human body that has many important functions such as prevention of water loss. Skin consists of two main structural layers: epidermis and dermis. Epidermis consists of 5 sublayers, the outer of which, stratum corneum (SC), is the main barrier that protects the body from dehydration, as well as from other molecules (e.g. irritants) entering the skin [8].

Dry skin is characterized by redness (erythema), scaling and, in extreme cases, even fissuring. Individuals with dry skin often complain about a burning sensation or itch. The water content at the outermost layer of SC is normally $15-20 \%$, in dehydrated or xerotic skin, on the other hand, it is only $10 \%$ [9]. Water content in SC is not homogeneous - it increases from around $15-30 \%$ at the outermost layer of $\mathrm{SC}$ to around $70 \%$ in the deeper living layer, and it stays at around $70 \%$ deeper in epidermis and dermis [10].

SC consists of dead keratinocytes also known as korneocytes (differentiated epidermal cells) that no longer possess a nucleus and are filled with keratin. Korneocytes also contain humectants or natural moisturizing factors (NMFs). These are molecules such as free amino acids and urea that are produced from a peptide called filaggrin. NMFs attract water molecules from dermal blood vessels to SC. This causes swelling of korneocytes, thus decreasing the inter-cellular space which is normally filled with hydrophobic lipids, e.g. ceramides, free fatty acids and cholesterol. This structure allows skin to keep the water inside the body, evading systemic dehydration. In addition, it does not allow toxic substances to penetrate the skin barrier. Transepidermal water loss (TEWL) is traditionally used to estimate the barrier function of the skin [11-12].

Application of moisturizing skin cosmetics reduces TEWL, maintains the physiological acidic $\mathrm{pH}$ level of skin surface and prevents degradation of intercellular connections and absorption of toxic substances. Skin moisturizers consist of three types of active substances: occlusives (petrolatum), humectants (urea, glycerin) and emollients (ceramides). Humectants attract water to SC from dermis; however, without the occlusive substances they could even increase TEWL. Occlusive moisturizers create a hydrophobic layer on skin surface that prevents the water that is attracted by humectants from leaving the corneal layer. Emollients are said to fill up the intracellular space in SC, thus reducing TEWL while also being less greasy than occlusive moisturizers [9].

\section{Materials and Methods}

In this study, we present a new experimental setup, as well as a new, more sensitive skin hydration parameter, compared to our previous work [7]. In addition, we did a clinical study in collaboration with Latvian cosmetics company MADARA. During this clinical study, we not only tested our developed experimental system based on NIR spectroscopy, but also compared results acquired by our system to the results acquired by a commercial device DermaLab (Cortex Technology, Denmark) that estimates skin hydration based on skin conductance measurements. In this study, we used DermaLab with a flat faced probe where the electrodes organized as concentric rings. The measurement range of DermaLab is 0-999 $\mu$ Siemens, $1 \mu$ Siemens resolution, 5\% accuracy. During the clinical study, we tested the shortterm (30 minutes) and long-term (1 month) effects of a new moisturizing cream developed by MADARA. The main components of the moisturizing cream are: Aqua/Water, Hippophae Rhamnoides (Sea-buckthorn) Fruit Extract, Sodium PCA, Urtica Dioica (Nettle) Leaf Extract, Linum Usitatissimum (Flax) Seed Extract, Paeonia Lactiflora (Peonia) Root Extract, Sodium Hyaluronate, and Hydrolyzed Hyaluronic Acid.

\subsection{Experimental setup}

Experimental setup consisted of a near-infrared spectrometer (NirQuest 512 by Ocean Optics) in the spectral range of 900-1700 nm, Y-type water-free optical fiber probe, and a stabilized tungsten-halogen light source (SLS201/M by Thorlabs) with radiation spectrum between 300 and $2600 \mathrm{~nm}$ (Fig. 1). This is a low-power light source (fiber-coupled optical power of $10 \mathrm{~mW}$ ) corresponding to approximately $1 \mathrm{~J} / \mathrm{cm}^{2}$ which is considered a low dose [13]. As one measurement usually took approximately 1 second at a time, we believe, it didn't significantly increase the temperature of skin surface or cause any other change in skin physiology. Optical fiber probe consisted of 6 illumination fibers (each $400 \mu \mathrm{m}$ in diameter) in a circle around 1 detection fiber $(400 \mu \mathrm{m}$ in diameter) without any separation. Due to very small separation between detection and illumination fibers $(0,4 \mathrm{~mm}$ between the fiber centers), we expected to detect diffuse reflected signal from mainly the superficial layers of skin (the upper sublayers of epidermis).

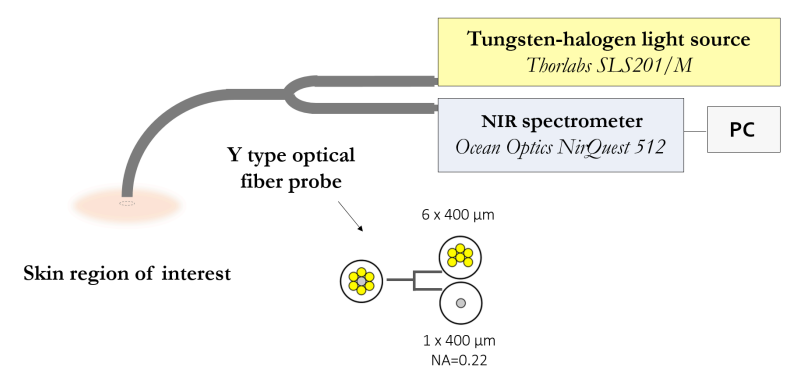

Fig. 1 Experimental setup for near-infrared diffuse reflectance measurements of skin.

Compared to the experimental setup we used in our previous study [7], in this setup the fiber probe end touched the surface of skin during the measurement. It is known that the compression of skin changes its optical properties [14-18]. As we were not able to include a pressure sensor in our experimental setup to determine the applied pressure for each measurement, the operator doing the measurements trained to apply approximately the same pressure for each measurement by just lightly touching the skin surface. In this way, we 
believe, the applied pressure to skin surface was very low and the variability of all measurements was minimized.

\subsection{Data analysis}

We measured reflected intensity from skin surface $I$ in the whole $900-1700 \mathrm{~nm}$ range by using the near-infrared spectrometer. We used a white reference tile Avantes $W S-2$ that reflects $98 \%$ of the radiation in the spectral range $350-1800 \mathrm{~nm}$ to measure illumination spectrum $I_{0}$; in this measurement, the fiber probe end was kept at a $5 \mathrm{~mm}$ distance from the reference tile. We then converted reflected intensity values to optical density (OD) values based on Beer-Lambert-Bouguer law. OD values represent absorption in tissue due to mainly water and lipids in this spectral range. Correction for dark current $\left(I_{\text {dark }}\right)$ was also applied:

$$
O D(\lambda)=\log _{10} \frac{I_{0}(\lambda)-I_{\text {dark }}(\lambda)}{I(\lambda)-I_{\text {dark }}(\lambda)} .
$$

A typical OD spectrum of skin in the spectral range of 900-1700 nm is shown in Fig. 2. There are three main absorption maxima: at around $980 \mathrm{~nm}, 1200 \mathrm{~nm}$ and $1450 \mathrm{~nm}$. The first two $(980 \mathrm{~nm}$ and $1200 \mathrm{~nm})$ are from a vibrational overtone of the O-H bond [19], while the maximum at around $1450 \mathrm{~nm}$ is due to the first overtone of the O-H stretching [20].

For estimation of skin hydration, we chose the water absorption maximum at $1450 \mathrm{~nm}$ as it has the highest water absorption in this range, thus it is more sensitive to small changes in water volume fraction, compared to the other maxima. Due to high absorption around the water absorption maximum at $1450 \mathrm{~nm}$, radiation around these wavelengths should not penetrate as deep in the tissue as other wavelengths in this spectral range, and in this study, we were mainly interested in the very upper sublayers of epidermis. We estimate that the radiation penetration depth around the water absorption maximum at $1450 \mathrm{~nm}$ is approximately $1 \mathrm{~mm}$ [21]. However, the measurement depth should be lower due to very small illumination and detection fiber separation (Fig. 1). Measurement depth of DermaLab, however, is much smaller - around $15 \mu \mathrm{m}$ [22].

To eliminate scattering effect on OD spectrum, we used linear baseline correction [23] between local minima at $1280 \mathrm{~nm}$ and $1650 \mathrm{~nm}$ (Fig. 2). After baseline correction, we calculated the volume area under the $1450 \mathrm{~nm}$ maximum which we chose as a parameter representing skin hydration.

\subsection{Clinical study}

We performed a clinical study to compare our developed system based on NIR spectroscopy and a commercial device DermaLab. In this clinical study, we tested the short-term (30 minutes) and long-term (1 month) effect of a moisturizing cream (developed by MADARA Cosmetics, Riga, Latvia) on skin hydration. In total, 39 volunteers (females, age range $18-60$, average: 45, standard deviation: 12) participated in this study. The study protocol was approved by the local ethics committee, and each participant gave a written informed consent before the trial. A primary screening procedure was done by a dermatologist before the study. Measurements were performed in January and February in Riga, Latvia with outside temperature around $15^{\circ} \mathrm{C}$ (January) and around $0{ }^{\circ} \mathrm{C}$ (February). During the measurements, room temperature was kept constant at around $23{ }^{\circ} \mathrm{C}$, and all participants were asked to sit at rest for 15 minutes at room temperature before the start of the measurements. All measurements were performed by two different devices - the developed device for estimation of skin moisture level by near-infrared spectroscopy, and a commercial device DermaLab which estimates skin hydration based on changes in skin conductance (with an increased water volume fraction in skin, there is an increase in electrical conductance of skin).

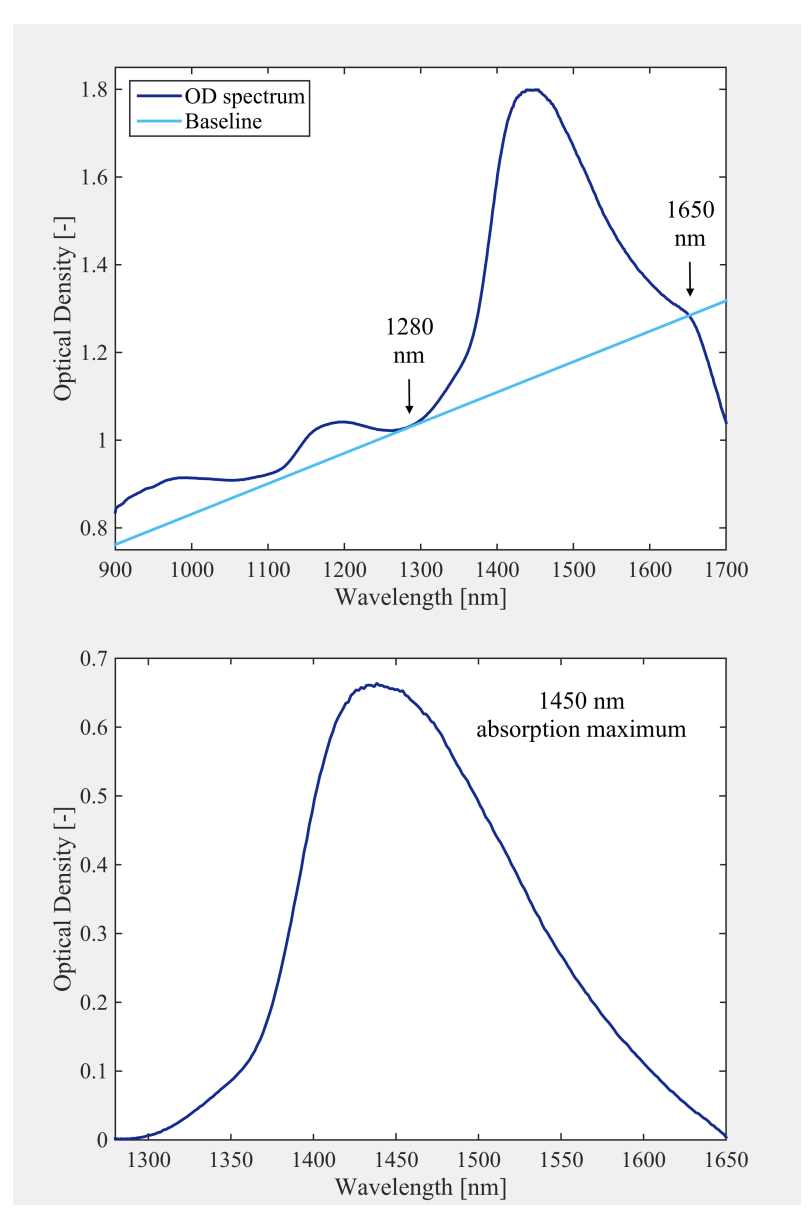

Fig. 2 Top: Example of an Optical Density (OD) spectrum of in vivo forearm skin and a baseline; Bottom: Baseline-corrected $1450 \mathrm{~nm}$ absorption maximum.

In the beginning, after the participant had sat still for at least 15 minutes, one measurement on right forearm (control) and three measurements on left forearm (Fig. 3 ) were performed by both devices. For the short-term (30 minutes) study, dermatologist applied the 
Table 1 Results of the change in skin hydration parameter of the short-term and long-term clinical study - comparison of both devices (NIR device and DermaLab).

\begin{tabular}{|c|c|c|c|c|c|c|c|}
\hline & & & NIR device & $\begin{array}{c}\text { Two-sample T } \\
\text { Test }\end{array}$ & & DermaLab & $\begin{array}{c}\text { Two-sample T } \\
\text { Test }\end{array}$ \\
\hline \multirow{4}{*}{$\begin{array}{c}\text { Short-term } \\
\text { study }\end{array}$} & \multirow{2}{*}{ Control } & $\Delta \mathrm{V}$ & $+1,4 \%$ & \multirow{2}{*}{$\begin{array}{c}\text { NO statistically } \\
\text { significant difference }\end{array}$} & $\Delta \mathrm{V}$ & $-5 \%$ & \multirow{2}{*}{$\begin{array}{c}\text { Statistically significant } \\
\text { difference }\end{array}$} \\
\hline & & SE & $0,7 \%$ & & SE & $1 \%$ & \\
\hline & \multirow{2}{*}{ Cream } & $\Delta \mathrm{V}$ & $+4,5 \%$ & \multirow{2}{*}{$\begin{array}{c}\text { Statistically significant } \\
\text { difference }\end{array}$} & $\Delta \mathrm{V}$ & $+7 \%$ & \multirow{2}{*}{$\begin{array}{c}\text { Statistically significant } \\
\text { difference }\end{array}$} \\
\hline & & SE & $0,6 \%$ & & SE & $1 \%$ & \\
\hline \multirow{4}{*}{$\begin{array}{l}\text { Long-term } \\
\text { study }\end{array}$} & \multirow{2}{*}{ Control } & $\Delta \mathrm{V}$ & $+2,3 \%$ & \multirow{2}{*}{$\begin{array}{c}\text { Statistically significant } \\
\text { difference }\end{array}$} & $\Delta \mathrm{V}$ & $+32 \%$ & \multirow{2}{*}{$\begin{array}{c}\text { Statistically significant } \\
\text { difference }\end{array}$} \\
\hline & & SE & $0,6 \%$ & & SE & $5 \%$ & \\
\hline & \multirow{2}{*}{ Cream } & $\Delta \mathrm{V}$ & $+1,9 \%$ & \multirow{2}{*}{$\begin{array}{c}\text { Statistically significant } \\
\text { difference }\end{array}$} & $\Delta \mathrm{V}$ & $+78 \%$ & \multirow{2}{*}{$\begin{array}{c}\text { Statistically significant } \\
\text { difference }\end{array}$} \\
\hline & & SE & $0,7 \%$ & & SE & $13 \%$ & \\
\hline
\end{tabular}

moisturizing cream on the proximal part of volar aspect of left arm. Afterwards, the participant was asked to wait for 30 minutes in a room at a temperature of $23{ }^{\circ} \mathrm{C}$, and another set of measurements were performed 30 minutes later - one measurement on right arm (control) and three measurements on left arm (where the cream had been applied). Three measurement points for left forearm were chosen due to possibly uneven application of the cream throughout the area. An average of the three measurements of left arm was later calculated and used for further analysis.

For the long-term study (1 month), participants were using the same moisturizing cream once a day on the same area of left forearm every day for one month. On the contrary, they were asked not to use any cream on the volar aspect of the right arm for the whole month. After 1 month, they came for another visit. After they had sat down still for at least 15 minutes to adapt to the room temperature, the same set of measurements was performed as in the beginning - one measurement on right arm (control) and three measurements on left arm where they had been applying the cream for the whole month.

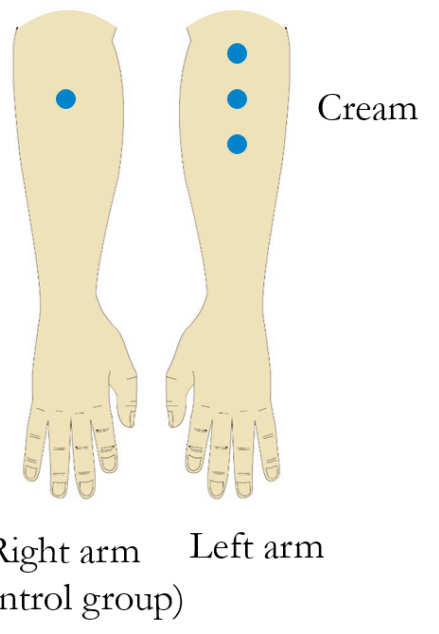

Fig. 3 Measurement points on the proximal part of volar aspect of right forearm (control group) and left forearm (cream group).

\section{Results and Discussion}

In results, separately for both devices, we calculated the increase (in percent) in skin hydration parameter 30 minutes/1 month later compared to the beginning of the study as the average for all 39 participants $(\Delta \mathrm{V})$, as well as the standard error $(S E)$. At first, we performed Lilliefors test in Matlab to test whether each measurement group (e.g. data of control group in the beginning of the study of all 39 participants) corresponds to normal distribution. Afterwards, a TwoSample T-Test was performed on two respective groups (e.g. control group in the beginning and control group 30 minutes later) to test whether there is a statistically significant difference between both groups, in other words, whether there is a statistically significant difference between results 30 minutes (or 1 month) later for all participants, compared to the beginning of the study. Results acquired by both devices are shown in Table 1.

$\Delta \mathrm{V}$ is the increase (in percent) in skin hydration parameter 30 minutes $/ 1$ month later compared to the beginning of the study as the average for all 39 participants, SE is standard error.

In this study, we used a near-infrared spectroscopy system for estimation of skin hydration in the spectral range of 900-1700 $\mathrm{nm}$ during a clinical study involving 39 female participants. We compared results acquired by our developed NIR device to results acquired by a commercially available device DermaLab that estimates skin hydration based on skin conductance measurements. We also tested the short-term (30 minutes) and long-term (1 month) effect of a moisturizing cream developed by MADARA Cosmetics on forearm skin.

Results of the short-term study are quite different when comparing both devices. In Table 1, we can see that there is no statistically significant difference between control group in the beginning and 30 minutes later, measuring by NIR device, which is what we expected. However, there is a statistically significant difference between control groups before and after, measuring by DermaLab device; there is even a $5 \%$ 
decrease in skin hydration parameter. On the other hand, results of both devices show a statistically significant increase in skin hydration parameter 30 minutes after application of the moisturizing cream: $+4,5 \%$ increase by NIR device and $+7 \%$ increase by DermaLab device. Even though the increase in percent is different for both devices, it is still comparable and shows the same trend for both devices.

Unexpectedly, results of the long-term study show statistically significant differences between both the control and the cream group by both devices. We expected an increase in skin hydration only for the cream group. These results suggest that there are other factors affecting skin hydration measurements, more so than the use of a moisturizing cream. Some of these factors might include the outside temperature (in the first set of measurements the outside temperature was around 15 degrees Celsius colder than in the second set of measurements one month later), use of different soap and/or other products on both forearms, physiological changes in skin etc. In future studies, when doing a long-term clinical study, measurements should preferably be done in similar external conditions such as room temperature and relative humidity.

We also tried to find a correlation between overall results by both devices. When comparing all patient data at the same temporal moment by both devices, we found very little correlation. Even though the trend is similar, at least for the short-term study (there is an increase in skin hydration 30 minutes after the cream was applied on skin surface), the results in general differ quite a lot between both devices. On one hand, it can be expected as both devices have different working principles and different measurement depths - while DermaLab measures skin hydration only in the SC (its measurement depth is around $15 \mu \mathrm{m}$ ), the measurement depth of NIR device is deeper and includes deeper sublayers of epidermis as well (possibly even upper dermis). After application of a moisturizing cream, water content is increased mainly in the SC (upper 10 $20 \mu \mathrm{m}$ in forearm skin), while it should not increase in deeper layers of epidermis. Because NIR device measures signal acquired from deeper epidermis, the increase of water content in the SC is less pronounced in the acquired overall signal. Therefore, DermaLab is expected to be more sensitive to even small changes in water content in SC.

The NIR device was developed in such a way to decrease the measurement depth as much as possible by arranging illumination and detection fibers without any separation between them. For analysis, only water absorption maximum of $1450 \mathrm{~nm}$ was used as it highly absorbs water, thus decreasing the penetration depth.
For future studies, cross polarizers might be incorporated in the experimental setup to reduce specular reflectance off the skin surface. Also, it might be useful to use illumination and detection fibers of a lower diameter (in this study, fibers of $400 \mu \mathrm{m}$ in diameter were used), thus possibly reducing the penetration depth even more.

Unfortunately, there is no "golden standard" method to estimate skin hydration and compare results of our developed device to. Even though there are different commercially available devices for estimation of skin hydration based on electrical properties of skin (conductance, capacitance), we believe that optical methods might be useful and maybe even better for this purpose as they can work in a non-contact mode (e.g. NIR imaging) providing less effect of skin surface nonuniformity and roughness, dependence on contact pressure on skin surface, compared to devices based on electrical properties of skin. During the measurements by DermaLab device, we noticed that the results vary quite a lot when doing a measurement at approximately the same spot of forearm skin within a short period (less than half a minute). In some test measurements, the standard deviation between 5 measurements on the same spot was close to $20 \%$. For NIR device, it was normally less than $2 \%$. We also noticed another issue regarding measurements by DermaLab device - for a couple of participants, skin hydration measurement 1 month later showed an increase of up to 10 times, compared to the beginning of the study, which seemed a very unlikely result. We considered these results as faulty and did not include them in the calculation of the average value. The reason might be the use of some specific chemical substances on skin that systems based on conductance measurements are very sensitive to, even though none of the participants could recall being exposed to anything unusual.

Overall, we conclude that DermaLab is more sensitive to small changes in skin hydration, compared to our developed NIR device. One of the reasons might be the deeper measurement depth by NIR spectroscopy device. There is currently no standardized method for NIR spectroscopy application for estimation of skin hydration, thus more research in this field is needed.

\section{Acknowledgements}

This work has been supported by Latvian National Research Programme SOPHIS under grant agreement no. 10-4/VPP-4/11 and by University of Latvia (project no. AAP2016/54). 